\title{
Self-organized $T_{e}$ redistribution during driven reconnection processes in high-temperature plasmas ${ }^{a)}$
}

\author{
H. K. Park ${ }^{\text {b) }}$ and E. Mazzucato \\ Princeton Plasma Physics Laboratory, Princeton, New Jersey 08543 \\ N. C. Luhmann, Jr., C. W. Domier, and Z. Xia \\ University of California at Davis, Davis, California 95616 \\ T. Munsat \\ University of Colorado at Boulder, Boulder, Colorado 80309
}

A. J. H. Donné, I. G. J. Classen, and M. J. van de Pol

FOM-Institute for Plasma Physics Rijnhuizen, Association EURATOM-FOM, P.O. Box 1207, 3430 BE Nieuwegein, The Netherlands

TEXTOR team

Forschungszentrum Jülich GmbH, Institut für Plasmaphysik, Association EURATOM-FZJ, D-52425 Jülich, Germany

(Received 5 December 2005; accepted 13 March 2006; published online 22 May 2006)

\begin{abstract}
Two-dimensional (2D) images of electron temperature fluctuations with high temporal and spatial resolution were employed to study the sawtooth oscillation in Toroidal EXperiment for Technology Oriented Research [S. S. Abdallaev et al., Nucl. Fusion 43, 299 (2003)] tokamak plasmas. The new findings are: (1) 2D images revealed that the reconnection is localized and permitted the determination of the physical dimensions of the reconnection zone in the poloidal and toroidal planes. (2) The combination of a pressure bulge due to finite pressure effects or a kink instability accompanied with a sharp pressure point leads to an " $X$-point" reconnection process. (3) Reconnection can take place anywhere along the $q \sim 1$ rational magnetic surface (both high- and low-field sides). (4) Heat flow from the core to the outside of the inversion radius during the reconnection time is through the finite opening on the poloidal and toroidal planes and the flow is highly collective. These new findings are compared with the characteristics of various theoretical models and experimental results for the study of the sawtooth oscillation in tokamak plasmas.

(C) 2006 American Institute of Physics. [DOI: 10.1063/1.2192467]
\end{abstract}

\section{INTRODUCTION}

The intrinsically enhanced energy confinement property of tokamak plasmas compared with other magnetic confinement devices is largely due to the rotational transform induced by a self-driven internal current. A common representation of the profile of the plasma current is via the so-called safety factor $(q)$ profile. Here, $q$ is simply a measure of the number of times a field line goes around a torus the long way (toroidal direction) for each time around the short way (the poloidal direction). In an ordinary tokamak plasma, the current-density profile is positive definite toward the center and the corresponding $q$ profile is monotonically increasing towards the edge. Under these conditions, the repetitive disruptive behavior of the plasma core within the $q \sim 1$ layer, commonly referred to as "sawtooth oscillation," was discovered in the early days of fusion plasma research. ${ }^{1}$ This is known as the $m / n=1 / 1$ internal kink mode where $m$ and $n$ are poloidal and toroidal mode numbers, respectively. An excellent review of recent research in the field of sawtooth oscillations is given in Ref. 2.

\footnotetext{
a) Paper GI1 4, Bull. Am. Phys. Soc. 50, 135 (2005).

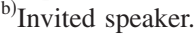

Understanding of the magnetic reconnection process associated with the sawtooth oscillation may provide insight into the global disruptive behavior of current-driven toroidal plasmas, where the nested magnetic field ruptures and the stored plasma energy instantaneously burst out to the periphery of the plasma. As a consequence, this understanding may contribute significantly to the success of next step magnetic fusion devices such as ITER (Ref. 3), which will be constructed by an international consortium. The topological change of the magnetic-field configuration, e.g., the magnetic reconnection process, is also a phenomenon of broad interest since it has been observed in laboratory plasmas, ${ }^{4}$ solar flares, ${ }^{5}$ and interstellar space. ${ }^{6}$ Many physical models developed to describe magnetic topology change in toroidally confined fusion plasmas, solar flares, and interstellar objects have actively contributed toward the goal of providing a common physics understanding of magnetic reconnection. In particular, the recent observation of sawtoothing radiofrequency (rf) emission from solar flares ${ }^{7}$ suggests that the physical mechanism of the repetitive small-scale disruptive behavior may be universal in current carrying toroidal plasmas.

The complexity of the phenomena requires sophisticated 
multidimensional diagnostic tools that can measure local physical quantities with high temporal and spatial resolution to precisely map out highly asymmetric physical phenomena like sawtooth oscillations in tokamak plasmas. This paper is the first such attempt to provide high spatial and temporal resolution 2D images of the highly asymmetric electron temperature fluctuation behavior obtained during the sawtooth oscillation. It reveals new experimental insights of the heat redistribution during the driven reconnection process which were not previously available. A brief background of theoretical and experimental studies of the sawtooth oscillation relevant to the experimental results is provided in Sec. II. In Sec. III, a description of 2D electron cyclotron emission (ECE) imaging on the Toroidal EXperiment for Technology Oriented Research (TEXTOR) (Ref. 8) device is provided. The detailed experimental observations of the reconnection processes such as " $X$-point" reconnection, observation of a high-field-side crash, and measurement of the toroidal localized reconnection zone, are discussed in Sec. IV. In Sec. V, a brief comparison of the experimental results with the relevant physical models is provided.

\section{BACKGROUND OF THE SAWTOOTH OSCILLATION AND DIAGNOSTIC TOOLS}

\section{Background of the sawtooth oscillation physics}

In the full reconnection model ${ }^{9}$ proposed by Kadomtsev and supported by simulation ${ }^{10}$ by Sykes, as the plasma current density in the core region increases $[q(0)$ drops below unity], the $m / n=1 / 1$ mode becomes unstable and a pressuredriven instability flattens the central pressure as well as the plasma current through an " $X$-point" reconnection zone along the magnetic pitch of the $q \sim 1$ surface on the reconnection time scale defined by earlier references. ${ }^{9,10}$ On the other hand, in the quasi-interchange model ${ }^{11}$ proposed by Wesson, the core plasma having a flat $q(q \sim 1)$ profile inside the inversion radius becomes unstable due to a slight change of magnetic pitch angle instead of a pressure-driven instability. As a result, the hot spot deforms into a crescent shape and cooler plasmas are convectively induced into the concave section of the crescent hot spot without any reconnection process. This model was supported by $\mathrm{x}$-ray tomography $^{12}$ on the Joint European Tokamak (JET) ${ }^{13}$ device; however, a later experimental study ${ }^{14}$ on the Tokamak de Varennas $(\mathrm{TdeV})^{15}$ device concluded that there exists no unique solution for the image through the inversion process of the chordal measurements of the x-ray tomography when only a limited number of independent viewing lines are used. The distinctively different evolution of the hot spot and/or cold island formation between the two models during the reconnection time could not be conclusively identified due to the lack of reliable 2D experimental tools. Furthermore, when no significant change was observed ${ }^{16,17}$ in the core plasma current $[q(0)$ was changed only from 0.7 to 0.8$]$ before and after the reconnection, both models were inconsistent with the fact that the value of $q(0)$ was less than $\sim 1$ before and after the reconnection. However, it should be noted that there exists an experimental result that $q(0)$ was changed from $\sim 0.7$ before the reconnection to $\sim 1.0$ after

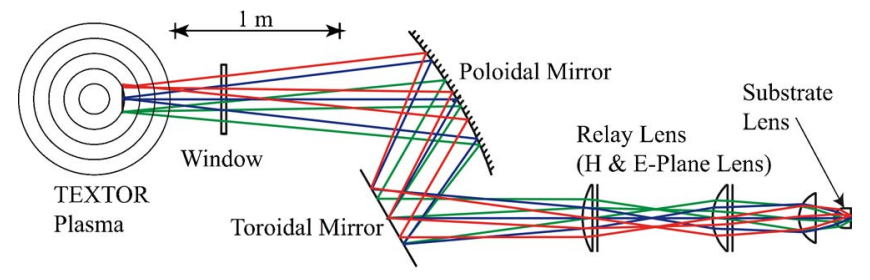

(a)

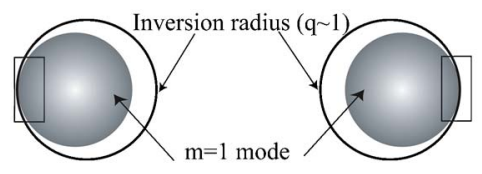

(b)

FIG. 1. Optical system design for the 2D ECE imaging system. (a) Large focusing mirrors (poloidal and toroidal) and relay lens (H \& E plane) are shown with the viewing plane and mapped image plane following the substrate lens. (b) Close views of the experimental arrangement for both the high-field (left) and low-field (right) sides for the imaging data to be discussed.

reconnection in a shaped plasma [DIII-D (Ref. 18)]. ${ }^{19}$ This could be an indication of differences in sawtooth stability between the circular and shaped plasmas. This discrepancy motivated further modeling of the driven reconnection process of the sawtooth oscillation such as a secondary reconnection process. ${ }^{20}$ Observation of a localized electron temperature bulge ${ }^{21,22}$ in the Tokamak Fusion Test Reactor $(\text { TFTR })^{23}$ device has been interpreted as a finite pressure effect on the sawtooth oscillation. ${ }^{24}$ Here, a pressure bulge of the $m / n=1 / 1$ mode at the low-field side leads to the global stochasticity of the magnetic field. ${ }^{24}$ This was thought necessary to reconcile a minute change of the current density and fast change of the pressure during the reconnection time observed in finite beta plasmas, where beta is the ratio between plasma pressure $\left(p=\Sigma_{j=i, e} n_{j} T_{j}\right)$ and the magnetic energy $\left(B^{2} / 2 \mu_{o}\right)$. The first pressure-driven ballooning mode instability was introduced to account for the observed disruptions in the high beta $\left[\beta_{p} \sim 1\right.$ and $\left.\beta_{\mathrm{t}}(0) \sim 4 \%\right]$ plasmas $^{25}$ in the TFTR device. These modes are more pronounced at the bad curvature side of the magnetic surface (low-field side in Fig. 1). Also, a three-dimensional (3D) local reconnection model where the reconnection zone is localized in the toroidal plane with many assumptions has been proposed in Ref. 21. In plasmas with a moderate beta $\left[\beta_{p}\right.$ $\sim 0.4$ and $\beta_{t}(0) \sim 1 \%$, where the present $2 \mathrm{D}$ imaging experimental results are to be discussed, the level of ballooning instability and global stochasticity of magnetic-field lines that is strongly coupled with the pressure surfaces, is moderate compared to those at high beta plasmas as demonstrated in Ref. 26.

The heat transport (redistribution) process during the sawtooth oscillation has also been extensively studied experimentally in order to understand the exceedingly fast time scale of the reconnection process of the sawtooth oscillations without precursors. ${ }^{22}$ In general, the time scale of the reconnection process is provided by Ref. 9 as 


$$
\tau_{c} \approx \frac{1}{2} \sqrt{\tau_{A}^{*}} \cdot \tau_{\eta},
$$

where $\tau_{A}^{*}$ is the modified Alfvén transit time and $\tau_{\eta}$ is the resistive diffusion time. Often, the "crash time" is referred to as the time period from the maximum value of $T_{e}(0)$ to the minimum value of $T_{e}(0)$, whereas the reconnection time is referred to as the time period from the time when island formation starts (indication of the reconnection at the lowerfield side) to the time when the island is fully stabilized. In general, the experimentally observed reconnection time is believed to be faster than the value given in Eq. (1). The faster time scale is often interpreted as the result of electron inertia, ${ }^{27}$ whereas the longer time scale is based on resistive behavior and is given in Eq. (1).

\section{Diagnostic tools for sawtooth oscillation study}

X-ray monitoring systems ${ }^{1}$ or $\mathrm{X}$-ray tomography ${ }^{12,14,28}$ have been routinely employed to study sawtooth oscillations in tokamak plasmas since their discovery. However, the $\mathrm{x}$-ray emission signal from hot plasmas is complex due to the multiple parametric dependence of the emission (electron density, temperature, and impurity content) and the limited number of chordal views in most cases hampers the determination of the uniqueness of each model. Even multiple views $^{28}$ around the torus can only provide a qualitative asymmetric view of the reconnection processes. In contrast, electron cyclotron emission, ${ }^{29}$ which depends solely on electron temperature in optically thick plasmas, ${ }^{30}$ has been a powerful tool to study local electron temperature fluctuations since the electron temperature is closely tied to the changes of the local plasma resistivity and known to be equalizing within the same flux zone on a thermal velocity time scale. However, ECE measurements have previously been limited to one dimension and reconstruction of the full image can only be achieved through reliance on symmetry arguments and various assumptions concerning the nature of the flux surface. Furthermore, knowing that the behavior of the electron temperature is highly asymmetric toroidally and poloidally on the reconnection time scale, it requires considerable imagination to reconstruct the heat redistribution process during the reconnection time scale. Recent studies of sawtooth physics at TEXTOR and Tore Supra ${ }^{31}$ using a onedimensional (1D) ECE system reported that the fast heat flow was also observed; however, it was not possible to study the topology of the reconnection process in detail.

\section{2D ELECTRON CYCLOTRON EMISSION IMAGING SYSTEM}

In magnetized plasmas, the electron gyro motion results in emission of radiation at the electron cyclotron frequency and its harmonics

$$
\omega_{c e}=\frac{e B}{m_{e}},
$$

where $B$ is the applied magnetic-field strength, $e$ is the electron charge, and $m_{e}$ is the electron mass. Diamagnetic and poloidal field effects are negligible in this experiment. In optically thick plasma where the electron density and temperature are sufficiently high, the radiation intensity approaches that of blackbody emission where the intensity is directly proportional to the local electron temperature. In tokamak plasmas, the ECE frequency has a spatial dependence due to the radial dependence of the applied toroidal magnetic field

$$
B(R)=\frac{B_{0} R_{0}}{R},
$$

where $R_{o}$ and $B_{o}$ are the geometric center and the magneticfield strength at the center of the plasma, respectively. The fundamentals of the ECE process are well established and have been routinely utilized to measure local electron temperatures in fusion plasmas. ${ }^{29,30}$ In a 2D electron cyclotron imaging (ECEI) system, the single antenna of a conventional $1 \mathrm{D}$ radiometer is replaced by an array of antennas. Figure 1(a) illustrates the optical design of the system where large collection optics and relay lens are required to project highresolution images of the local electron temperature onto sensitive 1D arrays through advances in array technology with well defined antenna patterns and state-of-the-art wideband rf and intermediate frequency (i.f.) electronics as described in detail in Ref. 32. A prototype system ${ }^{33}$ has been developed for the TEXTOR tokamak plasma. As shown in Fig. 1(b), the sampling volume of the image at the focal plane is $16 \mathrm{~cm}$ (vertical) $\times 8 \mathrm{~cm}$ (radial) for the experimental data from both high- and low-field-side views. The vertical resolution is determined by the antenna pattern of $\sim 2 \mathrm{~cm}$ [full width at half maximum (FWHM)] for each pixel and the radial resolution is $\sim 1 \mathrm{~cm}$ across the core of the tokamak plasma (total of 128 channels). The time resolution is primarily limited by the digitizer and the fastest time scale can be up to $5 \mu \mathrm{sec}$.

\section{EXPERIMENTAL RESULTS}

\section{Plasma parameters and sample 2D images}

The TEXTOR tokamak plasma has a circular shape with a major radius of $175 \mathrm{~cm}$ and a minor radius of $46 \mathrm{~cm}$. The range of toroidal magnetic field in the present work was in the range 1.9-2.4 $\mathrm{T}$ and the corresponding plasma current was $<305 \mathrm{kA}$. The $\mathrm{H}^{+}$plasma is heated with energetic neutral beams $\left(D_{0}, \sim 50 \mathrm{keV}\right.$, up to $\left.3 \mathrm{MW}\right)$ in order to maximize the temperature fluctuation of the sawtooth oscillation as well as to control plasma rotation (by varying the ratio of coinjection to counterinjection with respect to the direction of plasma current). The key plasma parameters were as follows: the central electron density and temperature range from 1.5 to $2.5 \times 10^{19} \mathrm{~m}^{-3}$ and from 1.2 to $1.6 \mathrm{keV}$, respectively. The corresponding peak toroidal beta is $\sim 1.0 \%$ and the average poloidal beta is between 0.3 and 0.5 . The toroidal rotation of the plasma varied from $\sim 1 \times 10^{4} \mathrm{~m} / \mathrm{s}$ to $\sim 8$ $\times 10^{4} \mathrm{~m} / \mathrm{s}$. The speed of a thermal electron is $\sim 6$ $\times 10^{7} \mathrm{~m} / \mathrm{s}$. The Alfvén and ion acoustic speeds are $5 \times 10^{6}$ and $7 \times 10^{5} \mathrm{~m} / \mathrm{s}$, respectively. Using plasma parameters close to the $q \sim 1$ surface, the calculated characteristic reconnection time $\left(\tau_{\mathrm{c}}\right)$ is $\sim 700 \mu \mathrm{s}$.

Since the radial coverage of the 2D ECEI system is limited to $\sim 7 \mathrm{~cm}$ near the core of the plasma, radial extensions 


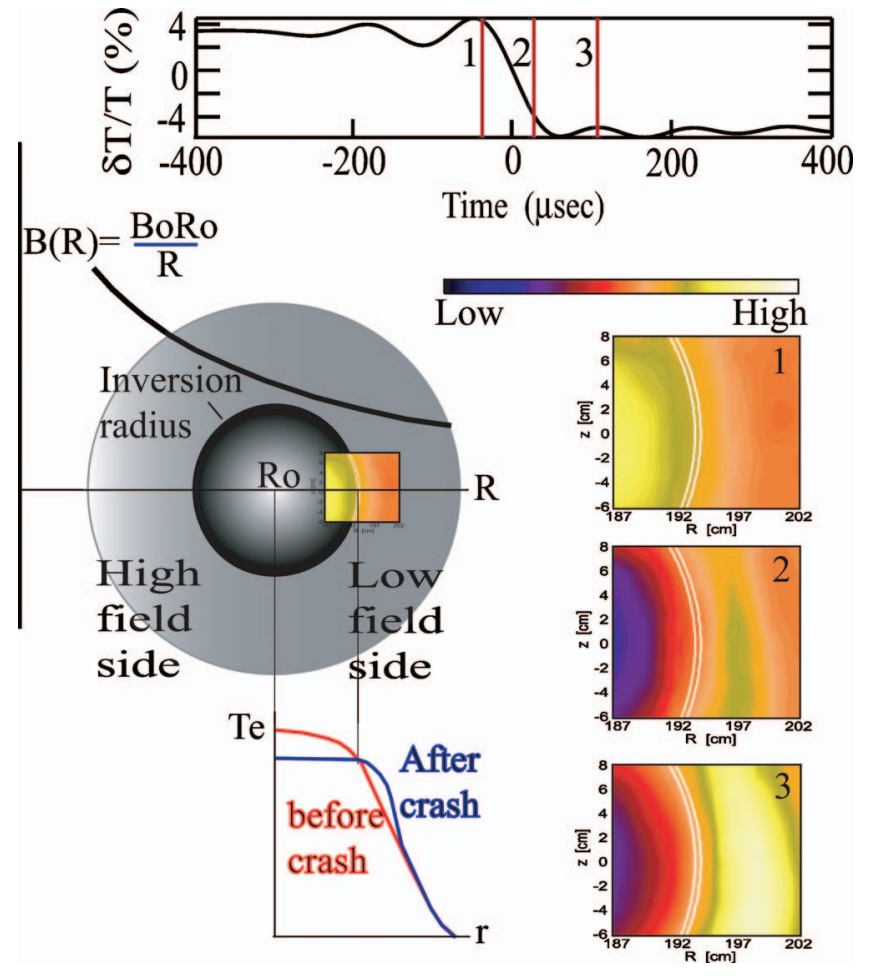

FIG. 2. (Color) The schematic of core temperature profile changes along with the poloidal cross section of the toroidal plasma and the time history of the electron temperature at $z=0$ and $R=187 \mathrm{~cm}$ are provided. 2D composite images of electron temperature fluctuations during the sawtooth crash phase are shown with the poloidal view of the plasma. (1) Partial image of the hot spot before the reconnection corresponds to a peaked profile of the electron temperature. (2) Transient image taken in the middle of the reconnection process. (3) Image after the reconnection is completed, corresponds to a flattened temperature profile.

of the image can be obtained with a variation of the LO source frequency and/or the applied magnetic field. The fluctuation quantities are relatively calibrated to the averaged value obtained with a long integration time and the intensity of the images is represented by $\delta T_{e} /\left\langle T_{e}\right\rangle$, where $T_{e}$ is the electron temperature, \langle\rangle is the time average, $\delta T_{e}$ is the fluctuation level $\left(=T_{e}-\left\langle T_{e}\right\rangle\right)$, and $\left\langle T_{e}\right\rangle$ is constant for the duration of many sawtooth oscillations. As demonstrated in previous ECE studies, the fluctuation level before and after the crash is introduced to emphasize changes of heat flow during the reconnection time. ${ }^{21}$

The measured 2D images of the electron temperature fluctuation through a composition of three images from discharges with slightly different magnetic fields $(2.3,2.35$, and $2.4 \mathrm{~T}$ ) are introduced to illustrate the reconnection process during sawtooth oscillation. Since the change of magnetic field was less than $\pm 2 \%$, there was little change in plasma parameters such as temperature and density. The composite image based on an average of $\sim 10$ identical sawtooth oscillations demonstrates that the behavior of the reconnection phenomena is consistent with classical pictures on a global scale as shown in Fig. 2. The portion of the $m / n=1 / 1$ mode (hot spot shown in whitish green color; frame 1) within the inversion radius (shown as a white double line) suddenly disappears and an island (cold spot shown as a bluish-black color) sets in, located in the zone previously occupied by the hot spot (frame 2). The heat removed from inside the inversion radius reappears at the outside of the inversion radius from the bottom of the low-field side and the heat distribution becomes symmetric (frame 3). The accumulated heat in the mixing zone eventually fades away through a diffusive process. The estimated inversion radius is solely determined from the temperature fluctuation data using the property that the relative variation of the temperature fluctuation is minimum near the inversion radius. Also, equilibrium analysis of the plasmas with similar plasma parameters indicates the $q$ $\sim 1$ is close to the inversion radius determined by this criterion. The actual transient reconnection physics, described below, will be addressed via images from a single frame image of the sawtooth oscillation.

\section{" $X$-point" formation during reconnection time at the low-field side}

There are many sawtooth oscillations in a single discharge (one sawtooth period is $\sim 15 \mathrm{~ms}$, whereas the discharge duration is $\sim 5 \mathrm{~s}$ ). After examination of many views of each sawtooth crash, a representative view of the sequence of $2 \mathrm{D}$ images during the reconnection process is provided in Fig. 3. Note that the phase of each reconnection process at the view position is strongly governed by the local nature of the reconnection event and the plasma rotation speed. The distortion of the $m / n=1 / 1$ mode at the low-field side is clearly illustrated after the symmetric image shown in frame 1 and the growth of a sharp temperature point shown in frames 3 and 4, which even crosses the inversion radius and leads to puncture of the magnetic surface as illustrated in this figure. The image of a sharp temperature formation is quite similar to the "pressure finger" of the model based on pressure-driven ballooning modes predicted to occur predominantly in the low-field side. The heat flow starts with a small opening and the opening grows as the heat flow increases to $\sim 15 \mathrm{~cm}$. The initial formation of the heat flow is similar to the " $X$ point" of the magnetic field as shown in frame 5. Since the time scale of the electron thermal velocity is ns and the progress of the image is tens of $\mu \mathrm{s}$, it is a reasonable assumption that the flow of the heat follows the local magnetic-field lines. The spatial broadening of the heat outside the inversion radius shown in frames 6 and 7 can account for the toroidal spread of the heat, since the transit time of electrons along the torus is on the order of a $\mu \mathrm{s}$. The flow of the heat through the opening of the magnetic field is highly collective which may not be the case if the global stochasticity in the later stage of the reconnection process is dominant. At moderate plasma beta values (poloidal and toroidal) similar to the experimental conditions, the growth of the ballooning mode and stochasticity is expected to be moderate. ${ }^{26}$ At the end of the reconnection phase, the temperature profile recovers symmetry (fully established island) as shown in frame 8 .

During examination of many reconnection images within the inversion radius from the rotating plasmas, it was found that the reconnection can happen everywhere along the poloidal plane in the low-field side as far as the $2 \mathrm{D}$ view is extended. Often, a portion of the images of the reconnection 

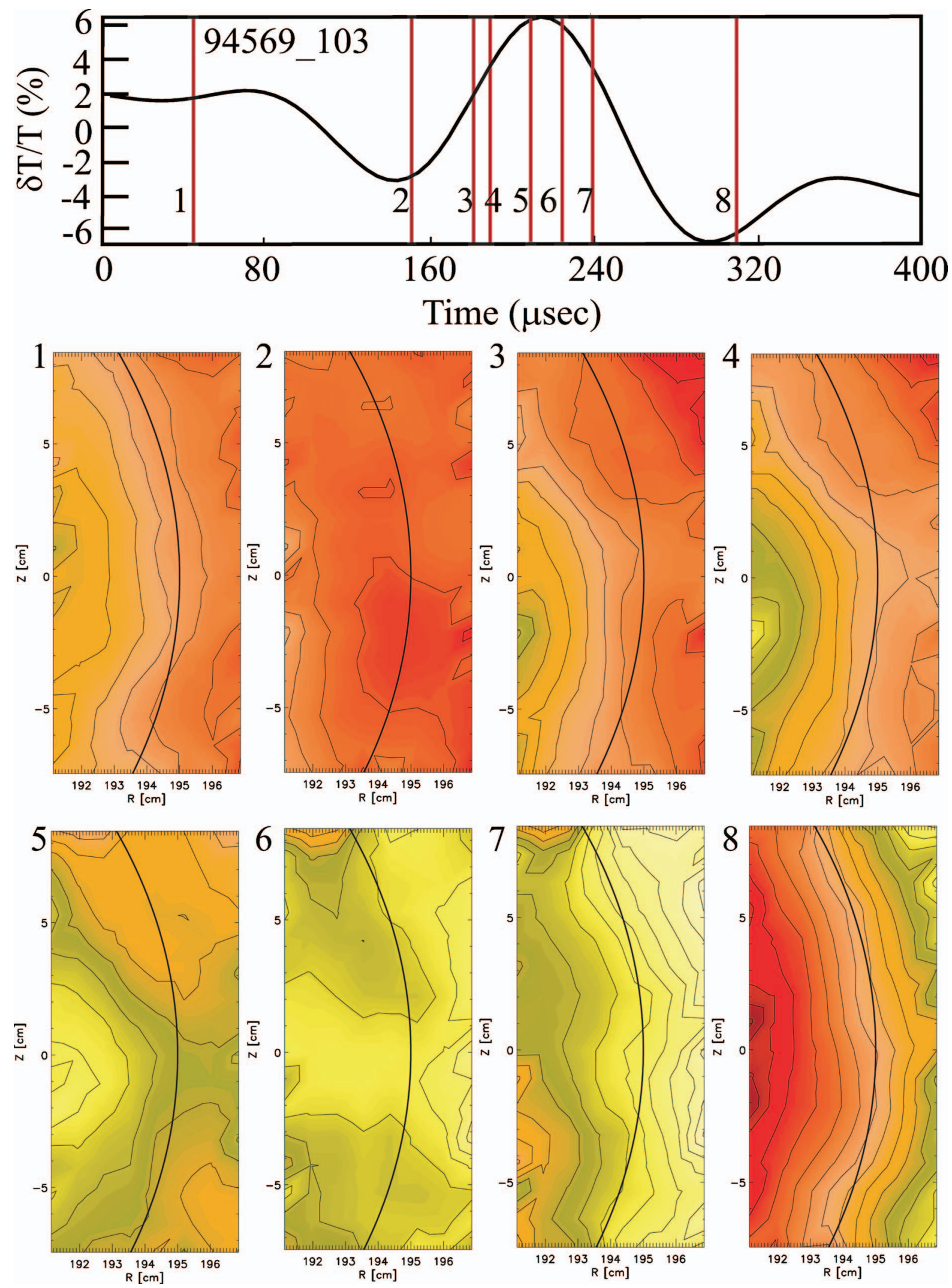

FIG. 3. (Color) 2D images of the sawtooth crash at the low-field side correspond to the times indicated in the electron temperature time trace at $z=0 \mathrm{~cm}$, $R=191 \mathrm{~cm}$. As the hot spot swells, as shown in frames 3 and 4, a sharp temperature point is growing and crosses beyond the inversion radius. Eventually, the temperature point leads to the reconnection. Initially it forms an " $X$ point" in the poloidal plane (frame 5) and heat starts to flow to the outside through a small opening. The heat flow is highly collective and the opening is increasing up to $\sim 15 \mathrm{~cm}$. At the end, the heat is accumulated outside the inversion radius and the poloidal symmetry is recovered. 

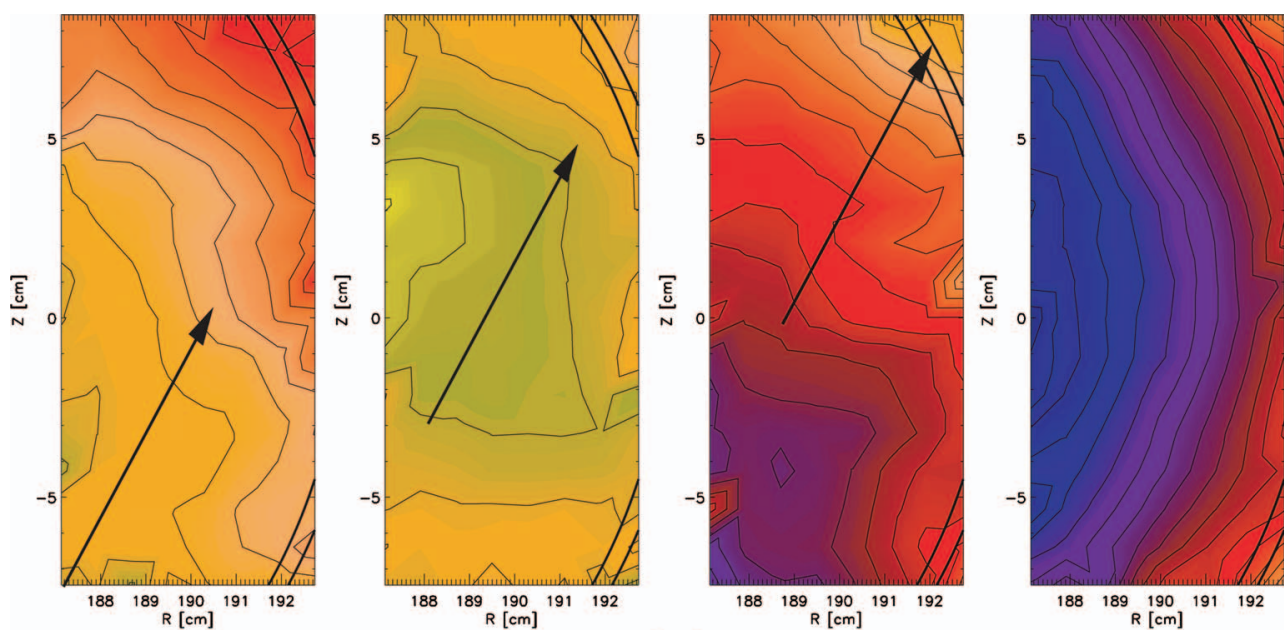

(a)
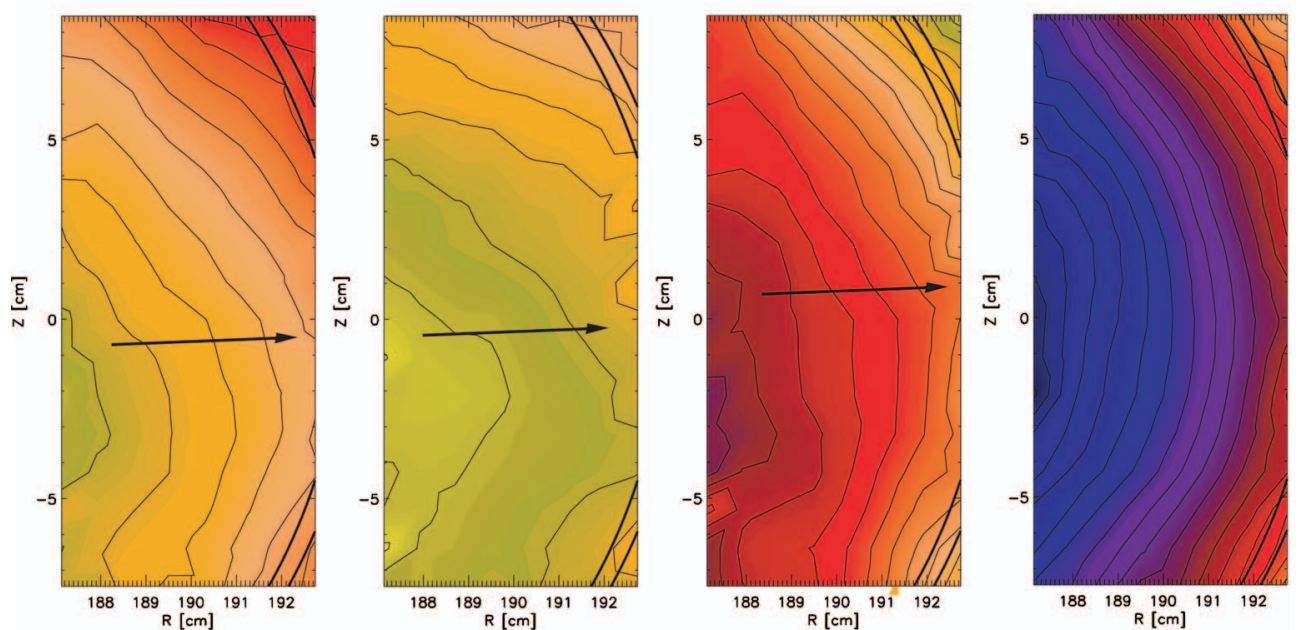

(b)
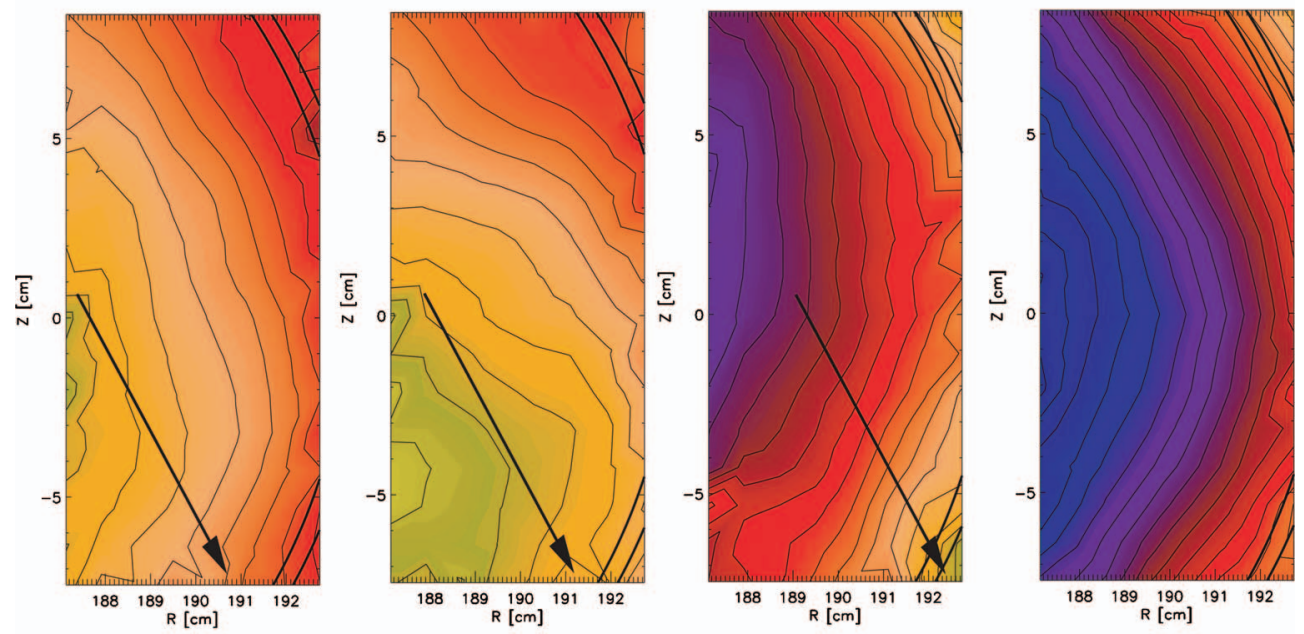

(c)

FIG. 4. (Color) Views of 2D images of the heat flow crossing the inversion radius (to the upper right-hand corner, to the midplane, and to the lower right-hand corner) at the low-field side are illustrated. 

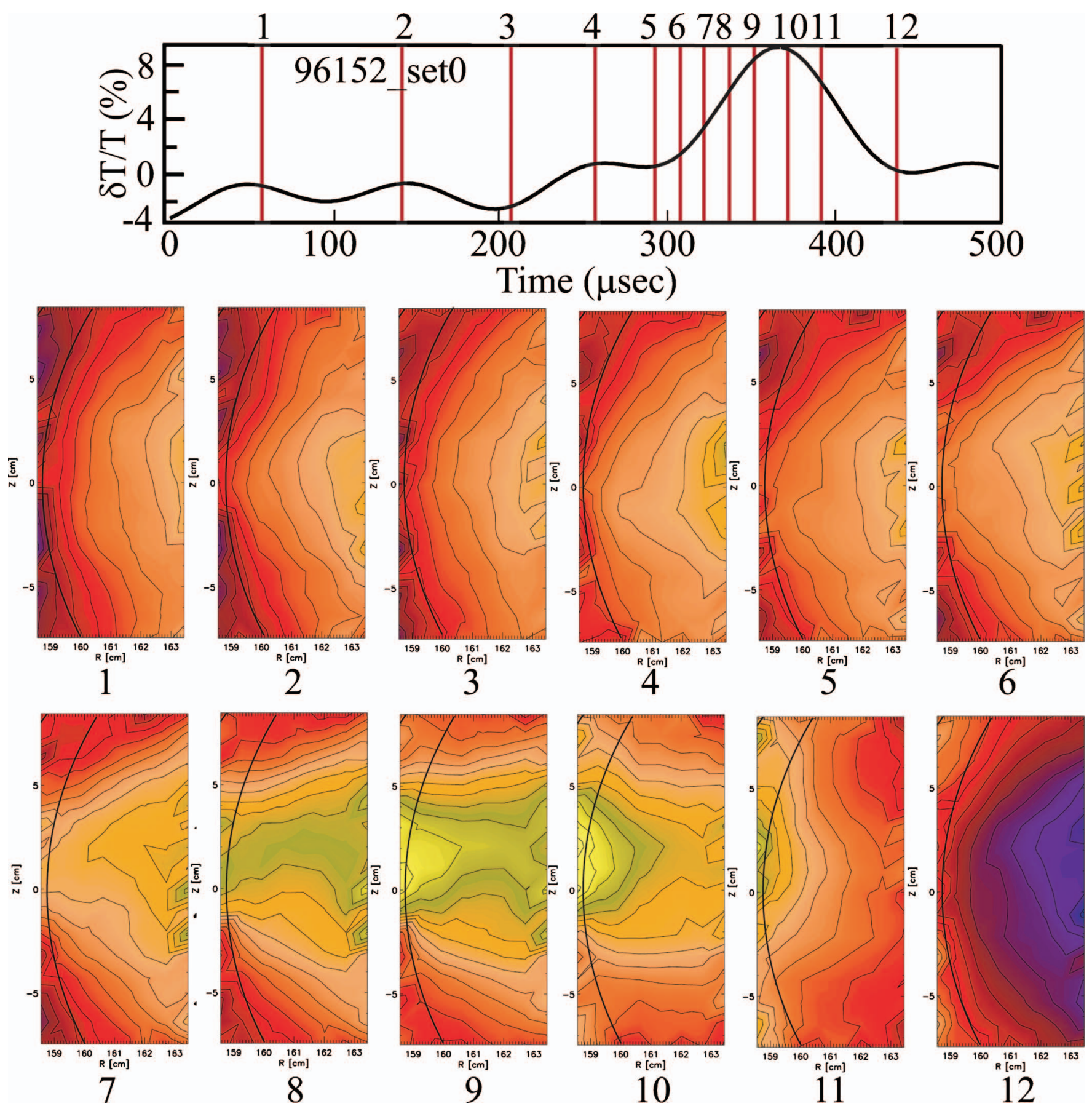

FIG. 5. (Color) 2D images from the sawtooth crash at the high-field side are shown with the time history of the electron temperature fluctuations at $z=0$, $R=148 \mathrm{~cm}$. The reconnection process is quite similar to that at the low-field side. A sharp temperature point develops with the moderate swelling of the hot core (frame 4), but fails to lead to reconnection in the first attempt. In the second attempt, the sharp temperature point accompanied with the strong swelling of hot spot (kink instability or finite pressure effect) (frames 6 and 7) succeeds in crossing the inversion radius through a small opening $(\sim$ a few $\mathrm{cm})$. The opening increases up to $\sim 10 \mathrm{~cm}$ and the heat flow is highly collective. The nested magnetic surfaces from the core push the heat flow out (frame 11) and eventually the symmetry is recovered (frame 12).

was captured near the top or bottom of the optical view located in the low-field side as shown in Fig. 4. In some cases, there is a sudden disappearance of the $m / n=1 / 1$ mode toward the center of the plasma and an island (cold) appears instead.

\section{D images of the sawtooth crash at high-field side}

In a second experimental campaign, the sample volume of the imaging system was moved to the high-field side of the $q \sim 1$ surface in order to explore reconnection phenom- ena there. Similar to the low-field-side case, many sawtooth oscillation images were reviewed. It was striking to find that the experimental results in the high-field side were quite similar to the observations made at the low-field side where the reconnection can happen everywhere along the poloidal plane. The progressive detailed images of the reconnection process at the high-field side are illustrated in Fig. 5. In the early stages, there is a symmetry of the $m / n=1 / 1$ mode as shown in frame 1 . In frame 2 , distortion of this mode is 
visible and a sharp temperature point is formed, but did not lead to the reconnection. This is attributed to the fact that the accompanying kink motion (swelling of the temperature) is not sufficiently strong to lead to reconnection. As the pointed surface is retreating, temperature swelling of the mode, which is indicative of the growing kink instability or pressure bulge due to the finite pressure effect, is shown in frames 3-6. In frame 7, the larger sharp temperature point pushes the magnetic surface beyond the inversion radius and heat flow starts through a small hole on the poloidal plane. The flow is highly collective as in the low-field side. The puncture in the poloidal plane begins with a small hole and grows to $\sim 10 \mathrm{~cm}$. As the heat gushes out, the nested field lines from the core (island) push the remaining heat from the rear. This is consistent with the flattening process of the pressure profile (current profile) in the full reconnection model. These processes are illustrated in frames $8-11$. As the heat is removed from the core, the closed field line topology is established and recovers the poloidal symmetry as shown in frame 12 .

\section{Toroidal extent of the reconnection zone}

The time history of one (innermost channel at $z=0$ ) of the 128 channels of the temperature fluctuation illustrates the typical precursor oscillations as the plasma rotates in the toroidal direction and the corresponding images at each time slice are shown in Fig. 6. The toroidal rotation speed is mainly driven by the momentum of the heating beam and the estimated speed is $\sim 6 \times 10^{4} \mathrm{~m} / \mathrm{s}$. The progressive swelling (or increase of the temperature fluctuation level) as the reconnection time is approached, as shown in frames 1 and 2 of the $m / n=1 / 1$ mode at the optical view position, is an indication of a kink instability as discussed in general magnetohydrodynamics (MHD) theory. ${ }^{34}$ The portion of this mode captured in each frame is quite symmetric as shown in these figures. The last symmetric partial view of this mode before the full crash (frame 2) is followed by the tail of the $m / n=1 / 1$ mode shown in frame 3 as it rotates away toroidally. As the plasma rotates further $\left(180^{\circ}\right.$ from frame 1$)$, the captured image contains a trace of island (cold spot) formation as shown in frame 4. There is no sign of the heat flow to the outside of the inversion radius even after the hot spot at the midplane of the low-field side passes to the opposite side $\left(180^{\circ}\right)$ from the view position. A hint of the heat leakage beyond the inversion radius has been detected when it rotates further (additional $90^{\circ}$ ) toward the optical view position and the heat flow pattern becomes highly asymmetric (frames 5-7). Reconnection is completed before the reconnection zone reaches the view position. At the end of the reconnection, the island is fully settled and symmetry is recovered as shown in frame 8 together with the island (cold spot). In this example, the reconnection process starts when the view of the mode at the low-field side on the midplane is around the $3 / 4$ turn from the last symmetric view (frame 2) and is completed just before it enters into the optical view. Using this information, the toroidal extent of the opening can be estimated and it is found to be roughly equal to $\sim 1 / 4$ of the toroidal circumference of the plasma $(\sim 2.5 \mathrm{~m})$.
A second example of the toroidal extent of the magneticfield puncture that can be estimated from the images of the sawtooth crash from rotating plasmas is given in Fig. 7, where the reconnection process starts from the bottom of the view and ends at the top of the view. The time required for the hot spot to cross the inversion radius is equivalent to the toroidal puncture size as shown in these images along the magnetic pitch of the $q \sim 1$ surface. Due to the fact that the poloidal opening of the magnetic surface is small (1/10 of the poloidal circumference) and the toroidal reconnection zone is along the magnetic pitch $(q \sim 1)$, both cases (Figs. 6 and 7) do not support the picture of localized reconnection in the toroidal direction. Often the hot spot suddenly moves toward the opposite direction of the low-field side even though it is expected to cross the inversion radius as illustrated in Fig. 7. Combining these observations with the randomness of the crash view within the inversion radius illustrated in Fig. 4, the toroidal extent of the reconnection zone has to be finite. Accurate estimation of the toroidal puncture size is limited due to the current size of the image and it is $\sim 3.3 \mathrm{~m}$ (larger than $1 / 4$ and less than $1 / 2$ of the toroidal circumference). A further point to note is that the toroidal reconnection zone is along the local magnetic pitch, since the captured images of the reconnection process vary with the phase of the crash at the view point.

The island formation starts in the early stage of the time histories of both examples shown in Figs. 6 and 7 and it takes some additional time for the island to relax after the crash. In the full reconnection model, the formation of the island is defined to be the beginning of reconnection since it is assumed that the formation of the island is the result of a topological change of the magnetic-field structure. The reconnection time, which starts when island formation is observed (precursor) and ends when the full island has been established in the core, is measured to be $\sim 500 \mu \mathrm{s}$, which is consistent with the value of the characteristic reconnection time $\left(\tau_{c}\right)$ provided in Eq. (1). However, it should be noted that the first hint of heat flow through the inversion radius is routinely observed in a later stage of the precursor. If the time when the first hint of heat flow through the inversion radius is observed is defined as the beginning time of the reconnection process rather than the time when the island is formed, the inferred reconnection time is $\sim 100 \mu \mathrm{s}$.

\section{COMPARISON BETWEEN THEORETICAL MODELS AND EXPERIMENTAL OBSERVATION}

High temporal and spatial resolution 2D images of electron temperature fluctuations during the reconnection time provide new insights into the heat transfer process during the driven magnetic reconnection process with a strong guiding magnetic field (i.e., the presence of a strong toroidal field in tokamak plasmas). The observed 2D images of the heat flow during the reconnection time exhibit a collection of bits and pieces of many theoretical models.

While the time evolution of the hot spot/cold island in the early stage of the sawtooth oscillation in Figs. 6 and 7 is strikingly similar to that expected from the full reconnection model, note that the reconnection process takes place later in 

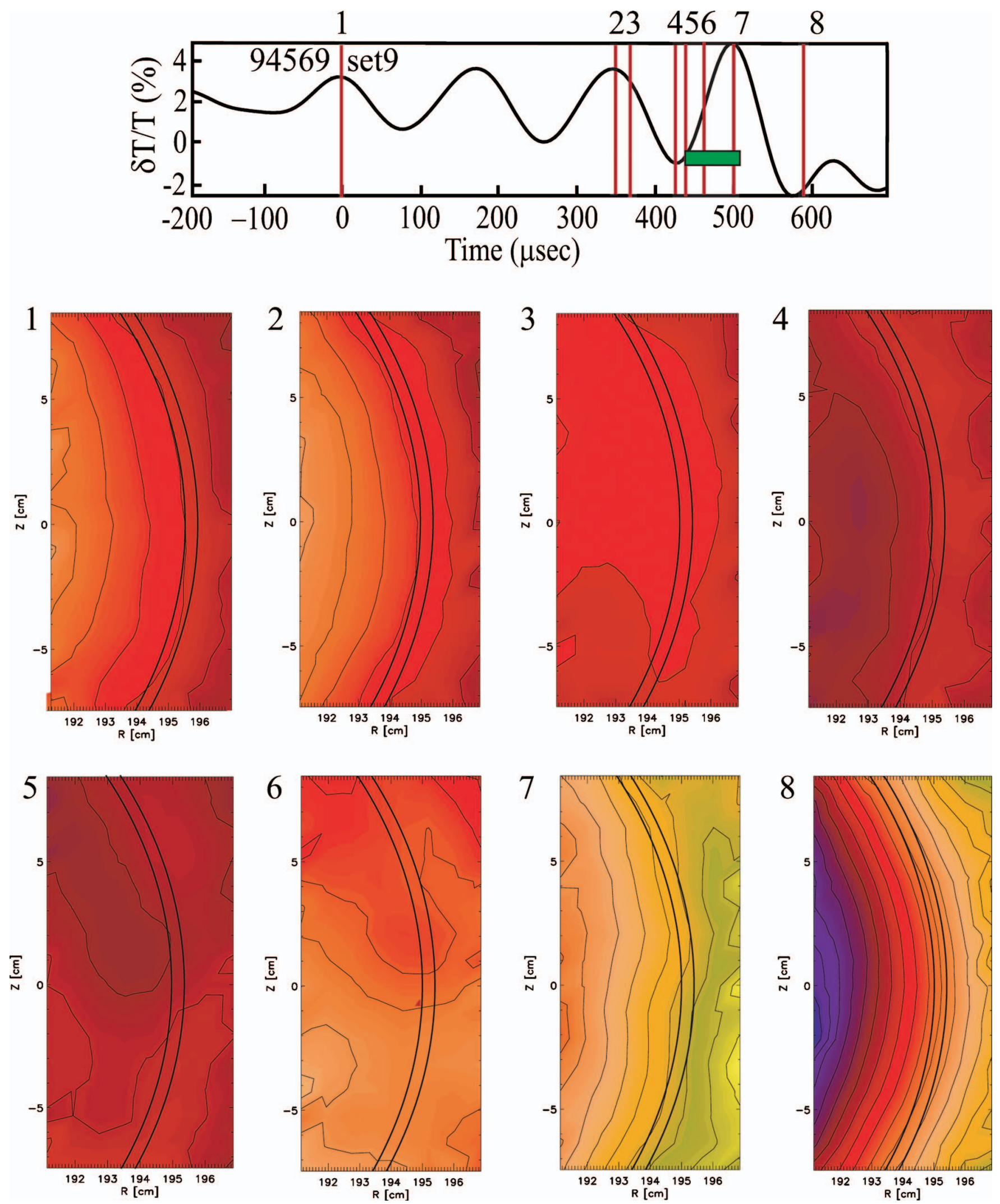

FIG. 6. (Color) Time series of high-resolution single frame image of the sawtooth crash evolution are illustrated. Direct view of the reconnection process was not accessible due to its localized nature. Swelling of the symmetric $m / n=1 / 1$ mode (frames 1 and 2 ) is an indication of temperature bulge due to the finite pressure effect or a kink instability. The toroidal extent of the reconnection zone $(\sim 2.5 \mathrm{~m})$ is estimated based the time scale of the heat leakage outside the inversion radius with the plasma rotation. 

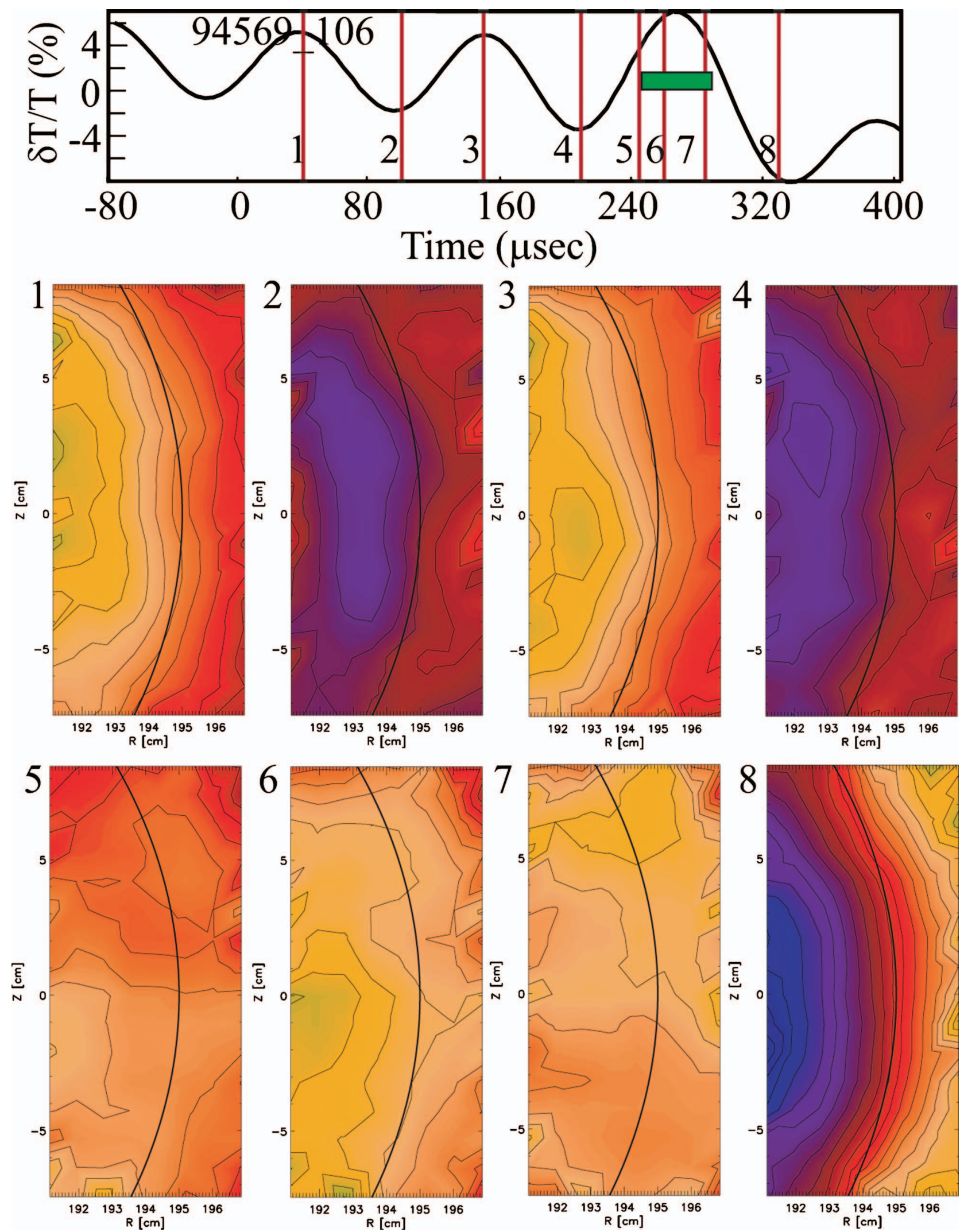

FIG. 7. (Color) Time series of high-resolution single frame image of the sawtooth crash evolution are illustrated. Here, a full view of the reconnection has been captured. Swelling of the symmetric $m / n=1 / 1$ mode (frames 1 and 3 ) is also visible. The toroidal extent of the reconnection zone ( $\sim 2.5 \mathrm{~m})$ is estimated based on plasma rotation. 
time. This observation suggests that there are additional physics mechanisms which may prevent the heat flow until a critical time. Alternatively, it could also imply that the reconnection process starts later in time, despite the fact that reconnection process is presumed to be initiated with the formation of the island. ${ }^{9}$ Observation of the finite extent of the toroidal reconnection zone ( $1 / 3$ of the toroidal circumference) is inconsistent with the full reconnection model where the plasma pressure and current density can be removed on a reconnection time scale given in Eq. (1) through a fully opened magnetic surface along the toroidal direction. However, the reconnection time given in Eq. (1) does not deviate from the experimental value based on the time scale of $\sim 500 \mu$ s that starts at the formation of the island and ends at the fully developed island formation. This is consistent with the observation that multiple toroidal views of x-ray tomography exhibit a fading hot spot away from the reconnection position. ${ }^{28}$ The role of the finite extent of the toroidal reconnection zone for the redistribution of the current density and pressure remains to be resolved in the future. The formation of a sharp point of the temperature perturbation at the lowfield side appears to be similar with the "pressure finger" of the ballooning model; however, the fact that the observed reconnection event has no preferential location along the poloidal magnetic surface at the moderate plasma beta may not be applicable to the ballooning model. ${ }^{25,26}$ The time evolution of the hot spot and island during the reconnection process is clearly inconsistent with that of the "quasiinterchange" model. The experimental observations suggest that the initial process is " $X$ point" and this is inconsistent with the model of " $Y$-point" formation at both separatrices of the island. ${ }^{26,35,36}$ The fact that heat flow from the core to the outside is through the finite opening of the magnetic field suggests that the global stochasticity of the magnetic-field lines may not be the dominant mechanism for this case. Also, the heat flow pattern appears to follow the local magneticfield lines and this observation may suggest that the pressure and magnetic-field lines are strongly coupled. The image of the " $X$-point" heat flow in the initial stage of the reconnection process initiated by a clear sharp temperature point accompanied with a strong kink or finite pressure effect (bulge of the $m / n=1 / 1$ mode) and collective heat transfer behaviors of the hot spot, resembles the hybrid reconnection process of the ballooning model ("pressure finger") followed by the full reconnection model.

\section{CONCLUSIONS}

Understanding of the driven magnetic reconnection process of the sawtooth oscillation is significant for many dynamic phenomena in plasmas confined by a magnetic field, such as the control of future generations of magnetic confinement devices and a better understanding of sawtooth phenomena in solar flares in which the guiding magnetic field is weak. A novel 2D ECEI system has been employed to capture the evolution of electron temperature variations during the reconnection time of the sawtooth oscillation in TEXTOR tokamak plasmas. A pressure-driven instability (sharp pressure point due to the distortion of the $m / n=1 / 1$ mode) accompanied by a kink instability or pressure bulge (due to the finite pressure effect on the $m / n=1 / 1$ mode) may be responsible for the magnetic reconnection process during the sawtooth oscillation. Only the evolution of the hot spot/ island before the reconnection and heat transport after the reconnection through the localized magnetic hole on the poloidal plane, resemble those of the full reconnection model. The observed pressure-driven sharp point of the temperature fluctuation at the low-field side is similar to the "pressure finger" of the ballooning mode model but the observation of the reconnection at both high- and low-field sides may not applicable to the finite pressure effect and/or ballooning mode model. The reconnection zone is localized along the torus $(1 / 10$ of the poloidal circumference and $1 / 3$ of the toroidal circumference).

\section{ACKNOWLEDGMENTS}

The authors are grateful to Dr. H. Soltwisch, Dr. E. Fredrickson, Dr. W. Park, Dr. M. Yamada, Dr. E. Westerhof, and Dr. H. de Blank for valuable discussions.

This work is supported by the U.S. Department of Energy under Contracts No. DE-AC02-76-CH0-3073, No. DEFG03-95ER-54295, and No. W-7405-ENG-48, and NWO and EURATOM.

${ }^{1}$ S. von Goeler, W. Stodiek, and N. Sauthoff, Phys. Rev. Lett. 33, 1201 (1974).

${ }^{2}$ R. J. Hastie, Astrophys. Space Sci. 256, 177 (1998).

${ }^{3}$ Y. Shimomura, R. Aymar, V. Chuyanov, M. Huguet, R. Parker, and ITER JCT, ITER Home Team, Nucl. Fusion 39, 1295 (1999).

${ }^{4}$ M. Yamada, Earth, Planets Space 53, 509 (2001).

${ }^{5}$ K. Shibata, Astrophys. Space Sci. 264, 129 (1999).

${ }^{6}$ D. Biskamp, Magnetic Reconnection in Plasmas (Cambridge University Press, New York, 2000), pp. 380.

${ }^{7}$ A. Klassen, H. Aurass, and G. Mann, Astro. Astrophys. 370, L41 (2001).

${ }^{8}$ S. S. Abdullaev, K. H. Finken, M. W. Jakubowski, S. V. Kasilov, M. Kovayashi, D. Reiser, A. M. Runov, and R. Wolf, Nucl. Fusion 43, 299 (2003).

${ }^{9}$ B. B. Kadomtsev, Sov. J. Plasma Phys. 1, 389 (1975).

${ }^{10}$ A. Sykes and J. A. Wesson, Phys. Rev. Lett. 37, 140 (1976).

${ }^{11}$ J. A. Wesson, Plasma Phys. Controlled Fusion 28, 243 (1986).

${ }^{12}$ R. S. Granetz et al., Nucl. Fusion 28, 457 (1988).

${ }^{13}$ J. Pamela, R. E. Solano, and JET EFDA Contributors, Nucl. Fusion 43, 1540 (2003).

${ }^{14}$ C. Janicki et al., Nucl. Fusion 30, 950 (1990).

${ }^{15}$ R. Decoste, B. L. Stanfield, J. L. Gauvreau et al., Plasma Phys. Controlled Fusion 38, A121 (1996).

${ }^{16}$ H. Soltwisch, Rev. Sci. Instrum. 70, 815 (1988).

${ }^{17}$ F. M. Levinton, L. Zakarov, S. H. Batha, J. Manickam, and M. C. Zarnstorff, Phys. Fluids B 5, 2554 (1993).

${ }^{18}$ V. S. Chen, C. M. Greefield, L. L. Lao, T. C. Luce, C. C. Petty, G. M. Staebler, and DIII-D team, Nucl. Fusion 40, 1137 (2000).

${ }^{19}$ B. W. Rice, D. G. Nilson, K. H. Burrell, and L. L. Lao, Rev. Sci. Instrum. 70, 815 (1999).

${ }^{20}$ D. Biskamp and J. F. Drake, Phys. Rev. Lett. 73, 971 (1994).

${ }^{21}$ Y. Nagayama, M. Yamada, W. Park, E. D Fredrickson, A. C Janos, K. M. McGuire, and G. Taylor, Phys. Plasmas 3, 1647 (1996).

${ }^{22}$ E. D. Fredrickson, K. McGuire, Z. Chang et al., Phys. Plasmas 2, 4216 (1995).

${ }^{23}$ R. J. Hawryluk, V. Arunasalam, C. W. Barns et al., Plasma Phys. Controlled Fusion 33, 1509 (1991).

${ }^{24}$ W. Park, D. A. Monticello, E. Fredrickson, and K. McGuire, Phys. Fluids B 3, 507 (1991).

${ }^{25}$ W. Park, E. D. Fredrickson, A. Janos, J. Manickam, and W. M. Tang, Phys. Rev. Lett. 75, 1763 (1995).

${ }^{26}$ Y. Nishimura, J. D. Callen, and C. C. Hegna, Phys. Plasmas 6, 4685 (1999). 
${ }^{27}$ J. A. Wesson, Nucl. Fusion 30, 2545 (1990).

${ }^{28}$ S. Yamaguchi, H. Igami, H. Tanaka, and T. Maekawa, Phys. Rev. Lett. 93, 045005 (2004).

${ }^{29}$ I. Hutchinson, Plasma Diagnostics (Cambridge University Press, New York, 1987).

${ }^{30}$ G. Bekefi, Radiation Process in Plasmas (Wiley, New York, 1966).

${ }^{31}$ V. S. Udintsev, M. Ottaviani, P. Maget et al., Plasma Phys. Controlled Fusion 47, 1111 (2005).
${ }^{32}$ H. Park, E. Mazzucato, T. Munsat et al., Rev. Sci. Instrum. 75, 3787 (2004).

${ }^{33}$ H. Park, C. C. Chang, B. H. Deng et al., Rev. Sci. Instrum. 74, 4239 (2003).

${ }^{34}$ G. Bateman, MHD Instabilities (MIT Press, Cambridge, MA, 1978).

${ }^{35}$ F. L. Waelbroeck, Phys. Fluids B 1, 2372 (1989).

${ }^{36}$ C. C. Hegna and J. D. Callen, Phys. Fluids B 4, 3031 (1992). 